URL www.una.ac.cr/mhsalud

\title{
EFECTO DEL MODELO "BAILE A LA SALUD", SOBRE EL NIVEL DE ACTIVIDAD FÍSICA, EL TIEMPO DEDICADO A MIRAR TELEVISIÓN, LA FRECUENCIA DE CONSUMO DE ALIMENTOS Y ESTADÍOS DE CAMBIO EN ADOLESCENTES MUJERES DE NOVENO AÑO
}

\author{
Óscar Hernández Quesada y Carlos Álvarez Bogantes ${ }^{2}$ \\ ${ }^{I}$ Ministerio de Educación Pública, Costa Rica \\ ${ }^{1}$ Universidad Nacional de Costa Rica \\ oshernandezque@hotmail.com
}

\section{RESUMEN}

\begin{abstract}
El objetivo de este estudio fue determinar la influencia de un programa de promoción de la salud llamado "Del Baile a la Salud" bajo el modelo Transteorético como base en la intervención baile latino (como forma de realización de actividad física) y charlas de alimentación y actividad física, sobre realización, tiempo dedicado a mirar televisión, frecuencia de consumo de alimentos y estadíos de cambio.

Participaron 66 mujeres adolescentes, 33 del grupo experimental y 33 del grupo control, con edades entre los 15 y 17 años, estudiantes de secundaria. Este programa tuvo una duración de 12 semanas, con una sesión de 80 minutos por semana. A esa población se le aplicó una evaluación inicial pretest, donde se obtuvo la cantidad en tiempo de la realización de actividad física (CAF), el tiempo dedicado a mirar televisión (TMT), Frecuencia de Consumo de Alimentos (FCA) saludables y no saludables (ENCA 1997-98) y la ubicación dentro de los estadíos de cambio (EC). Al finalizar, se realizó un postest con el objetivo de verificar cuáles fueron los cambios después de la aplicación del tratamiento.

Análisis estadístico: para determinar el efecto de la intervención en CAF, TMT, FCA y EC, se utilizó un ANOVA de dos vías, analizado por un paquete estadístico SPSS versión 10.

Resultados: con respecto a variable CAF del grupo experimental aumentó significativamente en minutos, entre tanto, el grupo control disminuyó su tiempo de realización de actividad física $(p<0.01)$. Por otro lado, en la variable TMT el grupo experimental disminuyó significativamente, en comparación con el grupo control, el cual aumentó el TMT ( $p<0.01)$. En el caso de EC los resultados fueron favorables en términos generales para el grupo experimental, a diferencia del grupo control cuyo comportamiento presentó tendencias negativas. Por último, en la variable FCA, no se observaron cambios significativos en el consumo promedio de los grupos de alimentos analizados.

Conclusiones: el programa logró crear cambios conductuales, en las variables de CAF, TMT, FCA y EC.
\end{abstract}

PALABRAS CLAVES: baile, modelo transteorético, adolescente.

\section{EFFECT OF A "DANCE TO HEALTH" MODEL ON THE LEVEL OF PHYSICAL ACTIVITY, TIME SPENT WATCHING TELEVISION, FOOD CONSUMPTION RATE, AND STAGES OF CHANGE IN FEMALE ADOLESCENTS IN NINTH YEAR}

\begin{abstract}
The aim of this study was to determine the influence of a health promotion program called "Dance to Health" under the Transtheoretical Model as a basis for intervention, Latin dance (as a form of physical exercise) and talks regarding food and physical activity on the time spent watching television, frequency of food consumption, and stages of change. A total of 66 female adolescents participated in the study; 33 were in the experimental group and 33 in the control group. Participants were high school students aged between 15 and 17. The program lasted 12 weeks, with one 80-minute session per week. An initial pretest was administered, which yielded the amount of time devoted to performing physical activity
\end{abstract}


URL www.una.ac.cr/mhsalud

(TPA), time spent watching television (TWT), food consumption rate (FCR), both healthy and unhealthy (ENCA 1997-98) and where the group was located within the stages of change (SC). At the end, a post-test was performed to verify the changes perceived after treatment. Statistical analysis: in order to determine the effect of the intervention on TPA, TWT, FCR, and SC, a two-way ANOVA was used and data was analyzed using the SPSS statistical package version 10. Results: regarding the TPA variable, the experimental group increased significantly in minutes, while the control group decreased the time used to perform physical activity $(p<0.01)$. On the other hand, in the TWT variable, the experimental group significantly decreased compared to the control group, which increased the TWT ( $p<0.01)$. In the case of SC, the results were generally favorable for the experimental group, unlike the control group whose behavior showed negative trends. Finally, in the FCR variable no significant changes were observed in the average consumption of food groups analyzed. Conclusions: The program was able to create behavioral changes in the TPA, TWT, FCR and SC variables.

KEY WORDS: dance, transtheoretical model, adolescent.

\section{INTRODUCCIÓN}

El ser humano ha sido creado para la realización de movimiento y la ejecución de actividad física, sin embargo esta se ha visto afectada por diversos avances tecnológicos que han creado una sociedad automatizada y han contribuido a un deterioro de la salud física y mental (Hoeger, Hoeger e Ibarra, 1996). Este fenómeno anteriormente descrito, ha sido observado en poblaciones jóvenes, ya que según Fernández et ál. (2001), indican que en la actualidad se ha logrado establecer que las y los niños y las y los adolescentes presentan una aptitud física deficiente, ya que no realizan la cantidad de actividad y de ejercicio físico necesaria para lograr beneficios en su salud, dedicando gran parte de su tiempo libre a la realización de actividades sedentarias tales como ver televisión, oír música, leer y juegos de video. Lo anterior también lo han comprobado autores como Balaguer, Pastor y Moreno (1999) y Avalos (2002), quienes encontraron una disminución en la cantidad de práctica deportiva y de actividad física conforme las y los adolescentes aumentan de edad, entre los 11 y los 17 años. Asimismo, Bianchi y Brinnitzer (2000), determinaron que cerca del 50\% del total de las alumnas encuestadas en su estudio, no practicaban actividad física fuera del contexto escolar.

De igual manera en Costa Rica se ha encontrado que un gran número de la población no practica ejercicio físico de la manera recomendada. En 1996 una encuesta determinó que un 53.4\% de la población de 12 a 70 años, nunca realizaba ejercicio físico contra un $2.9 \%$ que lo hace una vez al mes, $21.4 \%$ de una a dos veces a la semana y un $22.3 \%$ lo práctica durante tres o más ocasiones a la semana (Morice, 1998).

Y es que la poca realización de actividad física ocasiona estilos de vida sedentarios y esto se asocia a enfermedades, tal como lo presentan Monge, Muñoz, Figes, Rivera y Alvarado (1997), quienes señalan que entre un $52 \%$ y un $75 \%$ de población adolescente, presenta niveles altos de colesterol; por su parte Fernández y Moncada (2003), han determinado problemas de obesidad y sobrepeso en adolescentes costarricenses, ya que encontraron que entre un $15 \%$ y un $23 \%$ en la mujeres y entre un $14.9 \%$ y un $17.6 \%$ en hombres tienen sobrepeso y entre un $26 \%$ y un $5.8 \%$ en mujeres y de un $2.2 \%$ a un $5 \%$ de los varones tiene obesidad. Asimismo, la práctica de estilos poco saludables se ha podido observar hasta en la dieta del costarricense, ya que se encontró que el aporte calórico de la grasa en la dieta se incrementó del $20 \%$ en 1966 a $29.0 \%$ en 1991 y se mantiene en $27.4 \%$ en 1996. Por su parte, la ingesta de frutas y vegetales durante la adolescencia no alcanza los requerimientos mínimos de consumo, así lo reportó Monge (2001), donde indica que las y los adolescentes consumen $1.7 \%$ de frutas y $1.1 \%$ de vegetales.

Al contrario, la práctica de estilos saludables como la realización de la actividad física en la niñez y la adolescencia, puede generar una dosis de prevención para problemas del sistema cardiovascular, muscular, óseo y de enfermedades metabólicas como la diabetes (Fernández et ál., 2001). Estos 
URL www.una.ac.cr/mhsalud

hábitos de actividad física que se adquieren durante la niñez y la adolescencia, presentan una mayor probabilidad de mantenerse a lo largo de toda la existencia, proporcionando así la base de una vida activa y saludable (Organización Mundial de la Salud, 2001 y Organización Panamericana de Salud, 2000).

Estudios como el de Burke et ál.., (1998), Gortmaker et ál.., (1999), Harrell et ál.. (1999), han comprobado el éxito de la inserción de programas para mejorar la adopción de estilos de vida saludables en escolares con resultados positivos a corto, mediano y largo plazo, lo anterior concuerda con los objetivos de la Organización Mundial para la Salud (OMS) para la promoción de estilos de vida activos en las escuelas o los colegios, para aumentar la cantidad de actividad física (Organización Mundial para la Salud, 2001).

Una de las estrategias es el baile, ya que es una forma divertida de realizar actividad física que proporciona diversos beneficios fisiológicos propios del ejercicio aeróbico (Ainsworth et ál., 2000; Echegoyen, 2001; Monastra, 2002; Peidro et ál.., 2002), aunque es notable que el deporte ha sido más estudiado, tal vez porque la danza y el baile se han considerado como un arte, que no tiene nada que ver con la ciencia, se ha olvidado que hay una parte física que no se puede desligar del arte, no basta con la expresión, la danza se ha desarrollado tanto, que el bailarín ha rebasado las líneas de la normalidad corporal y trata de hacer proezas corporales no imaginadas; y es ahí en donde falta hacer ciencia (Echegoyen, 2001).

Por lo anterior, este estudio pretende determinar la influencia de un programa de promoción de la salud llamado "Del Baile a la Salud" bajo el modelo Transteorético, como base en la intervención baile latino (como forma de realización de actividad física) y charlas de alimentación y actividad física, sobre realización de actividad física, tiempo mirando televisión, estadíos de cambio y alimentación.

\section{METODOLOGÍA}

Sujetos: participaron 66 estudiantes mujeres adolescentes, con una edad promedio de 15 años. El grupo estaba dividido en dos: 33 grupo control y 33 grupo experimental. Distribuidas a conveniencia, ya que ambos grupos eran secciones previamente determinadas en el colegio.

Procedimiento: Se trabajó con dos grupos: el grupo control al cual no se le aplicó ningún tratamiento, reciben las lecciones regulares de Educación Física (Unidad de Baloncesto). Al grupo experimental se le aplicó el programa modelo del "Baile a la Salud".

En el desarrollo del mismo se utilizaron charlas y actividades de reforzamiento sobre la importancia de una adecuada alimentación y los beneficios del ejercicio físico. Este se aplicó bajo la utilización del Modelo Transteorético durante las lecciones de Educación Física que esta población recibía semanalmente (dos lecciones / 80 minutos por semana). A esa población se le aplicó una evaluación inicial pretest, donde se obtuvo la CAF, el TMT, la FCA y la EC. Al finalizar se realizó un postest con el objetivo de verificar cuáles fueron los cambios después de la aplicación del tratamiento.

Instrumentos: Se utilizó el cuestionario de "Recuento de Actividad Física del Día-Previo (Welk y Wood, 2000), para determinar la CAF y la TMT. En la evaluación del estado de alimentación de las adolescentes o FCA se utilizó la Encuesta Nutricional de Canarias (ENCA 1997-98), (Serra, Cabrera y Sierra, 2000), dándole énfasis en la ingesta de alimentos saludables y no saludables. Por último se utilizó el "Cuestionario de actividad física y estadíos de cambio" (Marcus y Forsyth, 
URL www.una.ac.cr/mhsalud

2003), para la ubicación de las adolescentes dentro de los diferentes estadíos de cambio, tanto al inicio de la intervención como al final de esta.

Análisis estadístico: se calcularon estadísticas descriptivas y un análisis de varianza de dos vías (mediciones por grupo), para determinar el efecto de la intervención en CAF, TMT, FCA y EC, todo mediante el paquete estadístico SPSS versión 10 para Windows.

\section{RESULTADOS}

Tabla 1. Resumen de promedios y desviaciones estándar del CAF (minutos en el día previo) según grupo y medición en mujeres adolescentes costarricenses.

\begin{tabular}{ccccc}
\hline & $\begin{array}{c}\text { Grupo Experimental } \\
(\mathrm{n}: 33)\end{array}$ & & \multicolumn{1}{c}{$\begin{array}{c}\text { Grupo Control } \\
(\mathrm{n}: 33)\end{array}$} & \multicolumn{1}{c}{$\begin{array}{c}\text { Total } \\
(\mathrm{n}: 66)\end{array}$} \\
\hline \multirow{2}{*}{ Pretest } & $54,54 \pm 31,33$ & Pretest & $49,09 \pm 42,30$ & 51,82 \\
Postest & $86,36 \pm 33,34$ & Postest & $36,36 \pm 42,92$ & 61,36 \\
\hline
\end{tabular}

*Según recuento de actividad física del día previo (Welk y Wood, 2000).

Tabla 2. Resumen de ANOVA mixto para el tiempo (minutos en el día previo) de actividad física según grupo y medición en mujeres adolescentes costarricenses

\begin{tabular}{cccc}
\hline Fuente & F & Sig. & Eta $^{2}$ \\
\hline MEDICIÓN & 8,233 & $0,006 *$ & 0,114 \\
MEDICIÓN * GRUPO & 44,826 & $0,000 *$ & 0,412 \\
GRUPO & 10,158 & $0,002 *$ & 0,137 \\
& & & \\
${ }^{*}<<0.01$ & & &
\end{tabular}

Gráfico1. Variable CAF para ambos grupos (minutos día previo)

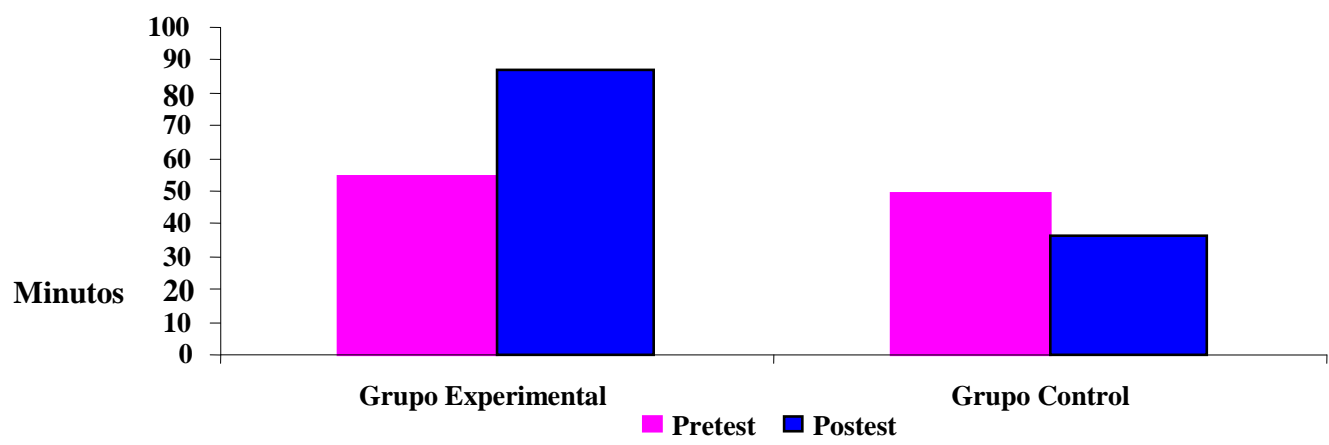


URL www.una.ac.cr/mhsalud

Tabla 3. Resumen de promedios y desviaciones estándar del TMT (minutos en el día previo) según grupo y medición en mujeres adolescentes costarricenses

\begin{tabular}{|c|c|c|c|c|c|c|}
\hline \multicolumn{3}{|c|}{$\begin{array}{c}\text { Grupo Experimental } \\
(\mathrm{n}: 33)\end{array}$} & \multicolumn{3}{|c|}{$\begin{array}{c}\text { Grupo Control } \\
(\mathrm{n}: 33)\end{array}$} & \multirow{2}{*}{$\begin{array}{c}\begin{array}{c}\text { Total } \\
\text { (n:66) }\end{array} \\
\pm 84,1178\end{array}$} \\
\hline Pretest & 135,4545 & $\pm 95,7892$ & Pretest & 117,2727 & $\pm 70,8993$ & \\
\hline Postest & 76,3636 & $\pm 65,8511$ & Postest & 130,6364 & $\pm 71,9652$ & $\pm 73,7034$ \\
\hline
\end{tabular}

*Según recuento de actividad física del día previo (Welk y Wood, 2000).

Tabla 4. Resumen de ANOVA mixto para la variable TMT (minutos en el día previo) según grupo y medición en mujeres adolescentes costarricenses

\begin{tabular}{ccll}
\hline Fuente & F & Sig. & Eta $^{2}$ \\
\hline MEDICIÓN & 8,964 & $0,004^{*}$ & 0,123 \\
MEDICIÓN * GRUPO & 22,504 & $0,000^{*}$ & 0,260 \\
GRUPO & 1,082 & 0,302 & 0,017 \\
& & & \\
\hline $\mathrm{p}<0,01$ & & &
\end{tabular}

Gráfico2. Variable TMT, grupo control y grupo experimental (minutos día previo).

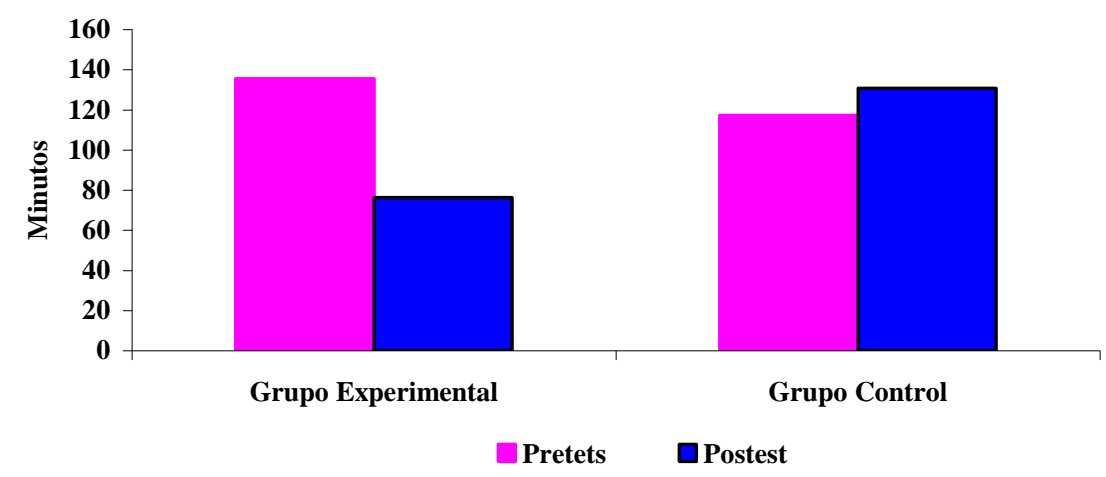

Como se aprecia en la tabla 1 y el gráfico 1, para el tiempo de realización de actividad física, se evidencia una interacción significativa. El análisis de efectos simples aplicado como Post Hoc, para el CAF, mostró que no existieron diferencias iniciales (pretest), entre ambos grupos, pero el grupo experimental aumentó significativamente su nivel de actividad física luego de la aplicación de la 
URL www.una.ac.cr/mhsalud

intervención, pasando de 54.54 minutos a 84.36 minutos. Entre tanto, el control pasó de 49.09 minutos a 36.36 minutos, siendo la diferencia de los tiempos promedio de actividad física en el postest significativa $(\mathrm{p}<0.01)$, entre ambos grupos.

Con respecto al TMT, el análisis de efectos simples mostró en la tabla 3 y en el gráfico 2, que igualmente no existieron diferencias iniciales en el pretest, entre ambos grupos, sin embargo el grupo experimental presentó una tendencia a disminuir significativamente el TMT (pretest 135.45 minutos, postest 76.36), mientras que el control aumentó su tiempo (pretest 117.27 minutos, postest 130.63 minutos). También se observa como en el post-test, las diferencias son significativas $(\mathrm{p}<0.01)$, entre el grupo intervenido y el grupo control.

En la evaluación de la frecuencia del consumo de alimentos, en el puntaje promedio de todos los grupos no se encontraron diferencias significativas. De tal manera que en el grupo de leche no se observaron diferencias significativas de grupo entre mediciones (mediciones F: 0,016; $>0,05$; grupo F: 1,079; p>0,05; medición por grupo F: 0,016; p>0,05). Es decir que los sujetos, tanto del grupo experimental como del control, no cambiaron entre mediciones su nivel de consumo de alimentos de este grupo.

Algo parecido se encontró para el grupo de grasas (mediciones F: 0,107; p>0,05; grupo F: 3,1; p>0,05; medición por grupo F: 1,144; p>0,05). También se obtuvo lo mismo con el grupo de carnes bajas en colesterol (mediciones F: 0,619; $>>0,05$; grupo F: 1,413; p>0,05; medición por grupo F: 0,019; $>$ > 0,05; grupo F: 0,032; p>0,05; medición por grupo F: 3,681; p>0,05). Así mismo ocurrió con el consumo de carnes altas en colesterol (mediciones F: 0,122; p>0,05; grupo F: 0,437; p>0,05; medición por grupo F: 0,17; p>0,05). Lo mismo se presentó para el consumo de alimentos de los cereales (mediciones F: 0.207: p<0.05; grupo F: 0.032; $\mathrm{p}<0.05$; medición por grupo F: 3.681 ; $\mathrm{p}<0.05)$. La misma situación se presentó para el consumo de alimentos de vegetales y frutas (mediciones F: 0,577; p>0,05; grupo F: 1,716; p>0,05; medición por grupo F: 0,33; p>0,05). Del mismo modo sucedió con el consumo de alimentos del grupo de los azúcares (mediciones F: 0,505; p>0,05; grupo F: 0,013; p>0,05; medición por grupo F: 2,679; p>0,05). Y lo mismo ocurrió con el consumo de comida chatarra (mediciones F: 0,166; p>0,05; grupo F: 1,576; p>0,05; medición por grupo $F: 1,77 ; p>0,05)$.

A continuación se presentan los resultados correspondientes a los estadíos de cambio de práctica de actividad física según grupo y mediciones, (tabla 5.a y 5.b). 
URL www.una.ac.cr/mhsalud

Tabla 5. Resumen de frecuencias absolutas y porcentajes por estadíos de práctica de actividad física según grupo y medición en mujeres adolescentes costarricenses.

\section{a. Grupo experimental}

Estadío de cambio post

Total

\begin{tabular}{|c|c|c|c|c|c|c|c|}
\hline Estadío de cambio pre & & $\begin{array}{c}\text { Pre } \\
\text { contemplación }\end{array}$ & Contemplación & Preparación & Acción & Iantenimiento & \\
\hline precontemplación & $\begin{array}{l}\text { Frecuencia } \\
\% \text { del total }\end{array}$ & 0 & $\begin{array}{c}1 \\
(3,0 \%)\end{array}$ & & & & $\begin{array}{c}1 \\
(3,0 \%)\end{array}$ \\
\hline contemplación & $\begin{array}{l}\text { Frecuencia } \\
\% \text { del total }\end{array}$ & 0 & $\begin{array}{c}5 \\
(15,2 \%)\end{array}$ & $\begin{array}{c}1 \\
(3,0 \%)\end{array}$ & $\begin{array}{c}10 \\
(30,4 \%)\end{array}$ & & $\begin{array}{c}16 \\
(48,5 \%)\end{array}$ \\
\hline preparación & $\begin{array}{l}\text { Frecuencia } \\
\% \text { del total }\end{array}$ & 0 & & $\begin{array}{c}1 \\
(3,0 \%)\end{array}$ & $\begin{array}{c}2 \\
(6,1 \%)\end{array}$ & & $\begin{array}{c}3 \\
(9,1 \%)\end{array}$ \\
\hline acción & $\begin{array}{l}\text { Frecuencia } \\
\% \text { del total }\end{array}$ & 0 & & & $\begin{array}{c}1 \\
(3,0 \%)\end{array}$ & $\begin{array}{c}5 \\
(15,2 \%)\end{array}$ & $\begin{array}{c}6 \\
(18,2 \%)\end{array}$ \\
\hline mantenimiento & $\begin{array}{l}\text { Frecuencia } \\
\% \text { del total }\end{array}$ & 0 & & & & $\begin{array}{c}7 \\
(21,2 \%) \\
\end{array}$ & $\begin{array}{c}7 \\
(21,2 \%) \\
\end{array}$ \\
\hline total & $\begin{array}{l}\text { Frecuencia } \\
\% \text { del total }\end{array}$ & 0 & $\begin{array}{c}6 \\
(18,18 \%)\end{array}$ & $\begin{array}{c}2 \\
(6,06 \%)\end{array}$ & $\begin{array}{c}13 \\
(39,39 \\
\%)\end{array}$ & $\begin{array}{c}12 \\
(36,36 \%)\end{array}$ & $\begin{array}{c}33 \\
(100,0 \%)\end{array}$ \\
\hline
\end{tabular}

\section{b. Grupo control}

Estadío de cambio post

Total

Estadío de cambio pre

Pre Contemplación Preparación Acción Mantenimiento

\begin{tabular}{|c|c|c|c|c|c|c|c|}
\hline contemplación & $\begin{array}{l}\text { Frecuencia } \\
\% \text { del total }\end{array}$ & $\begin{array}{c}2 \\
(6,1 \%)\end{array}$ & $\begin{array}{c}7 \\
(21,2 \%)\end{array}$ & & $\begin{array}{c}2 \\
(6,1 \%)\end{array}$ & & $\begin{array}{c}11 \\
(33,3 \%) \\
\end{array}$ \\
\hline preparación & $\begin{array}{l}\text { Frecuencia } \\
\% \text { del total }\end{array}$ & & $\begin{array}{c}2 \\
(6,1 \%) \\
\end{array}$ & & $\begin{array}{c}1 \\
(3,0 \%) \\
\end{array}$ & & $\begin{array}{c}3 \\
(9,1 \%) \\
\end{array}$ \\
\hline acción & $\begin{array}{l}\text { Frecuencia } \\
\% \text { del total }\end{array}$ & & $\begin{array}{c}1 \\
(3,0 \%) \\
\end{array}$ & & $\begin{array}{c}3 \\
(9,1 \%) \\
\end{array}$ & $\begin{array}{c}2 \\
(6,1 \%) \\
\end{array}$ & $\begin{array}{c}6 \\
(18,2 \%) \\
\end{array}$ \\
\hline mantenimiento & $\begin{array}{l}\text { Frecuencia } \\
\% \text { del total }\end{array}$ & & $\begin{array}{c}2 \\
(6,1 \%) \\
\end{array}$ & $\begin{array}{c}1 \\
(3,0 \%) \\
\end{array}$ & $\begin{array}{c}1 \\
(3,0 \%) \\
\end{array}$ & $\begin{array}{c}9 \\
(27,3 \%) \\
\end{array}$ & $\begin{array}{c}13 \\
(39,4 \%) \\
\end{array}$ \\
\hline Total & $\begin{array}{l}\text { Frecuencia } \\
\% \text { del total }\end{array}$ & $\begin{array}{c}2 \\
(6,1 \%)\end{array}$ & $\begin{array}{c}12 \\
(36,4 \%)\end{array}$ & $\begin{array}{c}1 \\
(3,0 \%)\end{array}$ & $\begin{array}{c}7 \\
(21,21 \%)\end{array}$ & $\begin{array}{c}11 \\
(33,33 \%)\end{array}$ & $\begin{array}{c}33 \\
(100,0 \%)\end{array}$ \\
\hline
\end{tabular}

Como se aprecia en la tabla 5.a, los resultados fueron favorables en términos generales para el grupo experimental. Solo un sujeto se encontraba en precontemplación en el pretest y logró pasar a contemplación en el postest. Así mismo de 16 sujetos que se encontraban en contemplación en el pretest, mejoraron su estadio ( 1 a preparación y 10 a acción). Del mismo modo, de 3 sujetos que se encontraban en preparación pasaron 2 a acción en el postest y de 6 que se encontraban en acción, 5 pasaron a mantenimiento en el postest. 
URL www.una.ac.cr/mhsalud

También se presentó el caso de sujetos que mantuvieron su mismo estadío entre pretest y postest, 1 en preparación, 1 en acción y 7 en mantenimiento.

En la tabla 5.b, se observa que el comportamiento del grupo control fue diferente al experimental y con tendencias negativas, pues a diferencia de este, en el grupo control se presentaron casos de sujetos que retrocedieron en su estadio ( 2 sujetos pasaron de contemplación a precontemplación, 2 sujetos pasaron de preparación a contemplación, 1 sujeto pasó de acción a contemplación, 2 sujetos de mantenimiento a contemplación, 1 sujeto pasó de mantenimiento a preparación y 1 sujeto pasó de mantenimiento a acción). También había sujetos que se encontraban en acción en el pretest y que se mantuvieron en ese estadio en el postest ( 3 sujetos) y 2 mejoraron a mantenimiento. Además, 9 sujetos en mantenimiento en pretest, mantuvieron ese estadio en el postest.

En términos generales se observa que los sujetos del grupo experimental tienden a mantener su estadio de práctica de actividad física (principalmente contemplación y mantenimiento) o a mejorar de estadio a acción. Mientras que el grupo control presenta algunos casos que mejoran de estadio, otros que lo mantienen (los que estaban en contemplación y mantenimiento principalmente), pero presenta muchos casos que empeoran su estadio.

\section{DISCUSIÓN}

El objetivo de este estudio fue determinar la influencia de un programa de promoción de la salud llamado "Del Baile a la Salud" bajo el modelo Transteorético como base en la intervención, baile latino (como forma de realización de actividad física) y charlas de alimentación y actividad física, sobre realización de actividad física, tiempo mirando televisión, estadíos de cambio y alimentación.

Se determinó que el grupo experimental aumentó el tiempo promedio de realización de actividad física luego de la intervención, obteniendo que un $84 \%$ de las adolescentes superan los 60 minutos requeridos por la OMS, contrario el grupo control el cual disminuyó el tiempo de esta variable pasando de un 55\% a un 33\% de adolescentes que efectuaban una hora o menos de actividad física. El hallazgo anterior es de suma importancia pues según Garita (2006), las mujeres adolescentes disminuyen la realización de actividad física por factores como la pérdida de feminidad y la discriminación asociada al deporte, elementos que influyen directamente en una población susceptible a desistir de los programas de ejercicio tradicionales pero que el baile probablemente influyó positivamente como lo indica Sanabria (2000). Lastimosamente, el sistema educativo nacional ha centrado la atención en la elaboración de planes donde se desarrolla el aprendizaje de destrezas deportivas, sin tomar en cuenta el elemento primordial para lograr adherencia a la realización de actividad física en las adolescentes; el gusto por determinada actividad física, lo cual a la postre como lo menciona Muñoz (2005) y se demostró en este estudio, pueden lograr cambios positivos en esta población.

\section{Variable de tiempo dedicado a mirar televisión:}

Según la OPS (2000), Rodríguez y Rondón (2000), Tercedor et al (2007), el tiempo dedicado a ver televisión ha aumentado con los años en esta población lo que podría generar deterioros en la salud de las personas vinculados a estilos de vida poco saludables y aparición de enfermedades. En este estudio se pudo encontrar modificaciones positivas en el tiempo que dedicaban las adolescentes a mirar televisión, ya que mientras el grupo experimental disminuyó el tiempo destinado a ver televisión, el grupo control lo aumentó. Eso podría generar una alternativa para combatir la problemática. 
URL www.una.ac.cr/mhsalud

\section{Variable de alimentación.}

Contrario a los resultados positivos que se encontraron en las variables anteriormente mencionadas, en el área de la evaluación de la alimentación no se hallaron cambios significativos en el consumo promedio de los grupos de alimentos analizados. Sin embargo, es importante rescatar que la ingesta del grupo de alimentos chatarra, tuvo un aumento en promedio tanto en el grupo experimental como en el grupo control, situación que a pesar de no ser significativa estadísticamente, no deja de ser alarmante, más aún, si esto lo respalda Suárez, Torrealba, Villegas, Osorio y García-Casal (2005), donde expone que las adolescentes presentan deficiencias de consumo en la mayoría de los grupos alimenticios. No obstante, se encontró una tendencia no significativa con la ingesta de frutas y vegetales, la cual aumentó de forma positiva en ambos grupos. Fontes de Oliveira y Kuhlmann, (2006), en su intervención igualmente con el Modelo Transteorético, sí encontró cambios estadísticamente significativos en el consumo de frutas y vegetales.

Los resultados encontrados en este aspecto pudieron deberse a que según Burke et al (1998), es de suma importancia la motivación de la familia al revisar sus dietas, de tal manera que se logre crear algún tipo de relación entre el hogar y la escuela. En el presente estudio se desarrollaron charlas específicas para las alumnas, dejando de lado la familia y por supuesto es en el hogar donde se preparan la mayor cantidad de alimentos que ellas consumen y aunque estas modifiquen su ingesta alimentaria durante el tiempo que permanecen en el colegio, en el hogar no es así. Por tal motivo se debe considerar incluir a los padres de familia dentro del proceso para poder crear modificaciones en el hogar y así reforzar el cambio en la alimentación de las adolescentes.

\section{Variable estadíos de cambio:}

En el caso de esta investigación se pudo observar modificaciones positivas en el postest del grupo experimental, por su parte en el grupo control se presentaron modificaciones negativas en algunos estadios. En este estudio un $75.7 \%$ se situó en el periodo de realización de actividad física de forma regular (acción y mantenimiento) muy distante a lo encontrado por Souza y Duarte (2005), en donde el $38.3 \%$ de los adolescentes practicaban actividad física regularmente (estado de mantenimiento). Siguiendo con lo citado por Souza y Duarte (2005), el 35.4\% hace actividad física irregularmente (estado de preparación), y el 26.2\% son sedentarios (precontemplación o contemplación). Este último dato difiere con lo obtenido por el grupo experimental al final de la aplicación del modelo, donde en el periodo de precontemplación, contemplación y preparación se reportó un $0 \%, 18.9 \%$ y $6 \%$ respectivamente, contrario al grupo control donde incluso el porcentaje en estado de no realización de actividad física (45.4\%), superó el promedio de lo presentado por estos autores; quienes además sugieren la creación de nuevas intervenciones en las escuelas, para así promover la práctica de actividad física, especialmente en las mujeres, y de buscar opciones que sean elegidas por ellas mismas, para aumentar la adherencia a la actividad física. Por su parte, Frenn y Malin (2003) y Woods, Mutrie y Scott (2002), también encontraron diferencias significativas $(\mathrm{p}<0,05)$ y cambios conductuales, respecto a los estadios de cambio de realización de actividad física utilizando Modelo Transteorético.

Por último, el baile ha sido mencionado por la Organización Mundial de la Salud como una actividad física agradable y divertida, donde el individuo puede expresarse y comunicar pensamientos y emociones (Jaramillo y Murcia, 2002; Sanabria, 2000), sin embargo en los programas de intervención aplicados por Harrel et al, (1999), (1996), Burke et al, (1998), Gortmaker et al, (1999) y Dunn et al, (1999), para crear modificaciones en los estilos de vida, han utilizado rutinas de ejercicio para inducir a la población a llevar estilos de vida más activos, sin 
URL www.una.ac.cr/mhsalud

tomar en cuenta la preferencia de la actividad, pero que sí fue considerado en este estudio, junto con charlas al igual que lo hicieron Harrel et al, (1999), Burke et al, (1998), Gortmaker et al, (1999) y Dunn et al, (1999). También este modelo se diferenció de los otros, en que la aplicación del tratamiento se presentó durante doce semanas, esto con el fin de aproximarse a una realidad trimestral del Modelo Educativo Nacional, diferente al tiempo utilizado por Gotmaker et al, (1999) y Harrel et al, (1996), quienes usaron programas de ejercicios con intervenciones de actividad física de tres veces por semana, durante ocho semanas. Las anteriores características pudieron haber contribuido en el éxito del programa.

Por último, el diseño de una intervención con un adecuado componente cognitivo, debe considerar la implementación de diferentes teorías como el Modelo Transteorético, todo con el propósito de aumentar la adherencia a estos programas y a la actividad física, siendo en este caso el modelo del "Baile a la Salud", una herramienta más, para la realización de actividad física, en las adolescentes, que puede crear modificaciones conductuales en los estilos de vida evaluados.

\section{BIBLIOGRAFÍA}

Ainsworth, B., Haskell, W., Whitt, M., Irwin, M., Swartz, A., Strath, S.,... León, A. (2000). Compendium of physical activities: an update of activity codes and MET intensities. Medicine \& Science in Sport \& Exercise, 32 (9), S498-S516.

Avalos, A. (2002). Malos Hábitos Enferman a Ticos. La Nación. www.lanación.com. Accesado el 10 de febrero, 2003.

Balaguer, I., Pastor, Y., y Moreno, Y. (1999). Algunas características de los Estilos de Vida de los Adolescentes de la Comunidad Valenciana. Revista Valenciana D'estudis Autonomics., (26), 33-56.

Bianchi, S., y Brinnitzer, E. (2000). Mujeres adolescentes y actividad física. Relación entre motivación para la práctica de la actividad física extraescolar y agentes socializadores. Revista digital Buenos Aires, 5(26).

Burke, V., Fracp, R., Thompson, C., Taggart, A., Dumbar, D., Spencer, M.,... Beilin, L. (1998). A controlled trial of health promotion programs in 11-year-olds using physical activity "enrichment" for higher risk children. The Journal of Pediatrics, 132(5), 840-848.

Dunn, A., Marcus, B., Kampert, J., García, M., Kohl III, H., y Blair, S. (1999). Comparison of Lifestyle and Structured Interventions to increase Physical Activity and Cardiorespiratory Fitness. The Journal of the American Medical Association. 281(4), 327-334.

Echegoyen, S. (2001). Cambios en el cuerpo del bailarín. Recuperado de:http://www.danzadance.org/bailetin/cambiosbailarin-echegoyen.html.

Fernández, A., González, C., Moncada, J., Pearson, G., Picado, M., y Salas, R. (2001). Normas Nacionales. Componentes de la salud física: Estudiantes Costarricenses entre 8 y 17 años. Costa Rica: Editorial Baula. 
URL www.una.ac.cr/mhsalud

Fernández, A., y Moncada, J. (2003). Obesidad y sobrepeso en la población estudiantil costarricense entre los 8 y 17 años. Revista Costarricense de Ciencias Médicas, 24(3-4), 95113.

Fontes de Oliveira, M., y Kuhlmann, G. (2006). The Transtheoretical model applied for fruit and vegetable consumption among adolescents. Revista de Nutrição, 19(1).

Frenn, M., y Malin, S. (2003). Diet and exercise in Low-Income Culturally Diverse Middle School Students. Public Health Nursing, 20(5), 361-368.

Garita, E. (2006). Motivos de participación y Satisfacción en la actividad física, el ejercicio físico y el deporte. Revista en Ciencias del Movimiento Humano y la Salud, 3(1).

Gortmaker, S., Cheung, L., Peterson, K., Chomitz, G., Hammond, J., Dart, H.,... Laird, N. (1999). Impact of school-based interdisciplinary intervention on diet and physical activity among urban primary school children. Archives of Pediatrics and Adolescent Medicine. 153(9), 975-983.

Harrell, J., McMurray, R., Gansky, S., Bangdiwala, S., y Bradley, C. (1999). A public health vs a risk-based intervention to improve cardiovascular health in elementary school children: the Cardiovascular Health in Children Study. American Journal of Public Health. 89(10), 15291535.

Hoeger, W., Hoeger., S. e Ibarra, G. (1996). Aptitud Física y Bienestar General. Tercera edición. Estados Unidos: Editorial, Morton Publishing Company.

Jaramillo, L., y Murcia, N. (2002). La Danza y el Baile. Revista Digital-EFDeportes. $8(46)$.

Marcus, B. y Forsyth, L. (2003). Motivating people to be physically active. United States of America: Human Kinetics.

Monastra, M. (2002). Programa de curso ritmos latinos. www.mauromonastra.com.ar/sitio/capacitacion.html.

Monge, R. (2001). Fruits and vegetables consumption among adolescents Costa Rica. Archivos Latinoamericanos de Nutrición, 51(1).

Monge, R., Muñoz, L., Figes, F., Rivera, A., y Alvarado J. (1997). Perfil lipídico de adolescentes urbanos costarricenses. Revista Costarricense de Ciencias Médicas, 18(2), 37-44.

Morice, A. (1998). Situación de las enfermedades crónicas no transmisibles en Costa Rica. http://www.inciensa.sa.cr/index.php?option=com_weblinks\&catid=49\&Itemid=263

Muñoz, S. (2005). La Educación Física escolar ante el problema de la obesidad y el sobrepeso. Revista Internacional de Medicina y Ciencia de la Actividad Física el Deporte, 5(19), 179-199.

Organización Mundial de la Salud. (2001). Estilos de vida sedentarios: un problema mundial de salud pública. www.who.int. 
URL www.una.ac.cr/mhsalud

Organización Panamericana de Salud. (2000). Análisis por género del comportamiento alimentario y el ejercicio físico en el Caribe. www.paho.org.

Peidro, R., Osses, J., Caneva, J., Brion, G., Angelino, A., Kerbage, S., García, M., y Pesce, R. (2002). Tango: modificaciones cardiorrespiratorias durante el baile. Revista Argentina de Cardiología, 70(5), 358-363.

Rodríguez, M. y Rondón, A. (2000). Hipercolesterolemia en la población adolescente. Revista de la Facultad de Medicina de Caracas Venezuela, 23(1), 50-4.

Sanabria, M. (2000). Bailoterapia: Diversión, salud y relax. www.seedo.es/actividadfisica.htm.

Serra, L., Cabrera, A., y Sierra, A. (2000). Conclusiones de la Encuesta Nutricional de Canarias (1997-98). Bases para una política de nutrición canarias. Archivos Latinoamericanos de Nutrición, 50(1), 62-70.

Souza, G. y Duarte, M. (2005). Estadíos de cambio de comportamiento relacionados a la actividad física. Revista Brasileira de Medicina do Esport, 11(2), 104-108.

Súarez, T., Torrealba, M., Villegas, N., Osorio, C., y García-Casal, M. (2005). Deficiencias de hierro, ácido fólico y vitamina B12 en relación a anemia, en adolescentes de una zona con alta incidencia de malformaciones congénitas en Venezuela. Archivos Latinoamericanos de Nutrició,. 55(2).

Tercedor, P., Martín-Matillas, M., Chillón, P., Pérez, I., Ortega, F., Wärnberg, J., Ruiz, J., y Delgado, M y grupo AVENA. (2007). Incremento del consumo del tabaco y disminución del nivel de práctica de actividad física en adolescentes españoles. Estudio AVENA. Nutrición Hospitalaria. 22(1), 89-94.

Welk, G. y Wood, K. (2000). Physical Activity Assessments in Physical Education. A practical Review of Instruments and their use in the Curriculum. The Journal of Physical Education, Recreation \& Dance, 71(1), 30-40.

Woods, C., Mutrie. N., y Scott, M. (2002). Physical activity intervention: a Transtheoretical Modelbased intervention designed to help sedentary young adults become active. Health Education Research, 17(4), 451-460.

\section{Agradecimientos}

Al Dr. Carlos Álvarez Bogantes, al M.Sc. Gerardo Araya Vargas y al M.Sc. Carlos Flores.

Fecha de recepción del artículo: 08 de febrero del 2010.

Fecha de aceptación del artículo: 14 de mayo del 2010.

Fecha de publicación del artículo: 31 de diciembre del 2010. 